\title{
Flow of MHD Carreau fluid in a curved channel
}

\author{
Saima Noreen ${ }^{\mathrm{a}, *}$, Tasawar Hayat ${ }^{\mathrm{b}}$ and Ahmed Alsaedi ${ }^{\mathrm{c}}$ \\ ${ }^{a}$ Department of Mathematics, Comsats Institute of Information Technology, Attock, Pakistan \\ ${ }^{\mathrm{b}}$ Department of Mathematics, Quaid-I-Azam University, Islamabad, Pakistan \\ ${ }^{\mathrm{c}}$ Department of Mathematics, Faculty of Science, King Abdulaziz University, Jeddah, Saudi Arabia
}

\begin{abstract}
Analysis has been made for the curvature effects on the MHD peristaltic flow of an incompressible Carreau fluid in a channel. The flow problem is first reduced in the wave frame of reference and then solved after employing the long wavelength and low Reynolds number approximations. Expressions of stream function, pressure gradient, magnetic force function, induced magnetic field and current density are derived and then examined for various parameters of interest.
\end{abstract}

Keywords: Curved channel, induced magnetic field, Peristaltic flow

\section{Introduction}

Peristalsis flows have vital role in physiology and engineering. Several authors have investigated such flows under various aspects since the seminal researches by Latham [1] and Shapiro et al. [2]. The studies on this topic have been undertaken both in the symmetric and asymmetric channels (see for example the attempts [3-10] and several refs. therein).

Presently the concept of magnetic field has importance in magnetotherapy, magnetic resonance imaging and magnetic devices. The motion of conductive fluid across the magnetic field induces currents, which thereby effects the propagating field. In fact the fluid flow is influenced by Lorentz force [11]. Motivated by such applications, some recent workers [12-17] in the field discussed the peristaltic flow in the absence of an induced magnetic field. On the other hand, Vishnyakov and Pavlov [18] investigated the peristaltic flow of viscous fluid in the presence of an induced magnetic field.

\footnotetext{
*Corresponding author: Saima Noreen, Department of Mathematics, Comsats Institute of Information Technology, Attock 43600, Pakistan. Tel.: +92 51 90642172, Fax: +92 51 2601171. E-mail: laurel_lichen@yahoo.com.
}

Mekheimer [19, 20] analyzed the induced magnetic field effects on flow of couple stress and micropolar fluid in a symmetric channel. Hayat et al. [21-25] put forward such analysis by examining the peristaltic transport of third order, fourth grade and Phan-ThienTanner fluids.

Peristaltic transport in curved channel is another important area of research which is not yet given a due attention. All the above mentioned studies have been undertaken in straight channels/tubes. Flow over curved channels is relevant to many industrial, biological and environmental applications. Examples are the spreading of pollution in a fjord and the wear experienced by pipes going through rough oceanic terrain. It is known that shape of most of the physiological tubes and glandular ducts is curved. Laser guiding in curved plasma channels have important applications such as an efficient circular x-ray laser medium, optical synchrotrons, laser accelerators and harmonic generators. Flow characteristics during flood associated with sediments transport is an application of curved geometry from engineering point of view. Micro heat exchangers involve curved channels where goal is often to increase the heat flux while limiting the pressure drop. 
Ali et al. [26] discussed the peristaltic flow of viscous fluid in a curved channel. Later on, they [27] extended the analysis by considering the heat transfer characteristics. Very recently Ali et al. [28] studied the peristaltic motion of third grade fluid in a curved channel.

This communication studies the induced magnetic field effects on the peristaltic flow of Carreau fluid in the curved channel. Section two deals with the modeling of governing equations. Solution to the problem and discussion are presented in the sections three and four respectively. Last section deals with the concluding remarks.

\section{Problem statement}

We consider a channel which is coiled just like a circle with radius $R^{*}$, half width $a$ and centre $O$ as shown in Fig. 1. An incompressible Carreau fluid fills the space in a curved channel. A sinusoidal wave of velocity $c$ and amplitude $b$ propagates on the flexible channel walls. The fluid in the channel flows due to the contraction and expansion of the walls of the channel. The inertial effects are taken small. We choose coordinates $(\bar{R}, \bar{X})$ with $\bar{X}$ in the direction of wave propagation and $\bar{R}$ transverse to it.

An external magnetic field of strength $H_{0}^{*}\left(=H_{0} \frac{R^{*}}{R^{*}+\bar{R}}\right)$ acts in the radial direction $\left(H_{0}\right.$ is the constant magnetic field). This results in an induced magnetic field $\mathbf{H}\left(\bar{h}_{\bar{r}}(\bar{R}, \bar{X}, \bar{t}), \bar{h}_{\bar{x}}(\bar{R}, \bar{X}, \bar{t}), 0\right)$ and therefore the total magnetic field becomes $\mathbf{H}^{+}\left(H_{0}^{*}+\bar{h}_{\bar{r}}(\bar{R}, \bar{X}, \bar{t}), \bar{h}_{\bar{x}}(\bar{R}, \bar{X}, \bar{t}), 0\right)$. The wall surface is described as follows:

$$
\bar{h}(\bar{X}, \bar{t})=a+b \sin \left(\frac{2 \pi}{\lambda}(\bar{X}-c \bar{t})\right) \text { upper wall, (1) }
$$

where $\lambda$ indicates the wavelength, $a$ the channel half width, $\bar{t}$ the time and $b$ the wave amplitude. If $\bar{V}$ and $\bar{U}$ are velocity components along the radial $(\bar{R})$ and axial directions $(\bar{X})$ respectively in the fixed frame, then the definition of velocity field $\mathbf{V}$ is

$$
\mathbf{V}=[\bar{V}(\bar{R}, \bar{X}, \bar{t}), \bar{U}(\bar{R}, \bar{X}, \bar{t}), 0]
$$

The present flow situation is governed by the following fundamental equations:

$$
\nabla \cdot \mathbf{V}=0
$$

$$
\begin{aligned}
\rho \frac{d \mathbf{V}}{d t} & =\operatorname{div} \mathbf{T}+\mu_{e}\left(\nabla \times \mathbf{H}^{+}\right) \times \mathbf{H}^{+} \\
& =\operatorname{div} \mathbf{T}+\mu_{e}\left[\left(\mathbf{H}^{+} \cdot \nabla\right) \mathbf{H}^{+}-\frac{\nabla \mathbf{H}^{+^{2}}}{2}\right] \\
\frac{d \mathbf{H}^{+}}{d t} & =\nabla \times\left(\mathbf{V} \times \mathbf{H}^{+}\right)+\frac{1}{\varsigma} \nabla^{2} \mathbf{H}^{+} \\
\nabla \cdot \mathbf{E} & =0, \quad \nabla \cdot \mathbf{H}=0 \\
\nabla \times \mathbf{E} & =-\mu_{e} \frac{\partial \mathbf{H}}{\partial t}, \quad \nabla \times \mathbf{H}=\mathbf{J} \\
\mathbf{J} & =\sigma\left(\mathbf{E}+\mu_{e}(\mathbf{V} \times \mathbf{H})\right) .
\end{aligned}
$$

In the above equations $\varsigma=\sigma \mu_{e}$ denotes the magnetic diffusivity, $\sigma$ the electrical conductivity, $\mu_{e}$ the magnetic permeability, $\rho$ the density, $d / d t$ the material derivative and $\mathbf{J}, \mathbf{E}$ and $\mathbf{H}$ as the current density, the electric field and the magnetic field respectively.

The Cauchy stress tensor $\mathbf{T}$ in Carreau fluid is

$$
\begin{aligned}
& \mathbf{T}=-\bar{p} \mathbf{I}+\bar{\tau} \\
& \bar{\tau}=-\left[\eta_{\infty}+\left(\eta_{0}-\eta_{\infty}\right)\left(1+(\Gamma \overline{\dot{\gamma}})^{2}\right)^{\frac{n-1}{2}}\right], \\
& \overline{\dot{\gamma}}=\sqrt{\frac{1}{2} \sum_{i} \sum_{j} \overline{\dot{\gamma}}_{i j} \overline{\dot{\gamma}}_{j i}}=\sqrt{\frac{1}{2} \Pi=\sqrt{\frac{\operatorname{trac} A_{1}^{2}}{2}}}
\end{aligned}
$$

where $p$ is the pressure, I the identity tensor, $\bar{\tau}$ the extra stress tensor, $\eta_{\infty}$ the infinite shear rate viscosity, $\eta_{0}$ the zero shear-rate viscosity, $\Gamma$ the time constant, $n$ the dimensionless power law index, $\Pi$ the second invariant of strain-rate tensor and Equation (10) for $\eta_{\infty}=0$ becomes

$$
\begin{aligned}
& \bar{\tau}=-\left[\eta_{0}\left(1+(\Gamma \overline{\dot{\gamma}})^{2}\right)^{\frac{n-1}{2}}\right] \overline{\dot{\gamma}}, \\
& \overline{\mathbf{A}}_{1}=\overline{\mathbf{L}}+\overline{\mathbf{L}}^{T}, \quad \overline{\mathbf{L}}=\operatorname{grad} \overline{\mathbf{V}},
\end{aligned}
$$

and for $n=1$ or $\Gamma=0$ we have the case just like viscous fluid.

In fixed frame we have the following set of equations

$$
\frac{\partial}{\partial \bar{R}}\left\{\left(R+R^{*}\right) V\right\}+R^{*} \frac{\partial U}{\partial X}=0,
$$




$$
\begin{aligned}
& \rho\left[\frac{\partial \bar{V}}{\partial t}+V \frac{\partial \bar{V}}{\partial \bar{R}}+\frac{R^{*} \bar{U}}{R^{*}+\bar{R}} \frac{\partial \bar{V}}{\partial X}-\frac{\bar{U}^{2}}{R^{*}+\bar{R}}\right] \\
& =-\frac{\partial \bar{p}}{\partial \bar{R}}+\frac{1}{R^{*}+\bar{R}} \frac{\partial}{\partial \bar{R}}\left\{\left(R^{*}+R\right) \bar{\tau}_{\bar{R} \bar{R}}\right\} \\
& +\frac{R^{*}}{R^{*}+\bar{R}} \times \frac{\partial \bar{\tau}_{\bar{X}} \bar{R}}{\partial \bar{X}}-\frac{\bar{\tau}_{\bar{X} \bar{X}}}{\bar{R}+R^{*}}-\frac{\mu_{e}}{2 \rho}\left(\frac{\partial H^{+^{2}}}{\partial \bar{r}}\right) \\
& +\frac{\mu_{e}}{\rho} \times\left[\left(H_{0} \frac{R^{*}}{R^{*}+\bar{R}}+\bar{h}_{r}\right) \frac{\partial \bar{h}_{r}}{\partial R}\right. \\
& \left.+\bar{h}_{\bar{x}} \frac{R^{*}}{R^{*}+\bar{R}} \frac{\partial \bar{h}_{r}}{\partial X}-\frac{\bar{h}_{x}^{2}}{R^{*}+\bar{R}}\right], \\
& \rho\left[\frac{\partial \bar{U}}{\partial t}+\bar{V} \frac{\partial \bar{U}}{\partial \bar{R}}+\frac{R^{*} U}{R^{*}+R} \frac{\partial U}{\partial X}-\frac{U V}{R^{*}+R}\right] \\
& =-\frac{R^{*}}{R^{*}+\bar{R}} \frac{\partial p}{\partial X} \\
& +\frac{1}{\left(R^{*}+R\right)^{2}} \frac{\partial}{\partial R}\left\{\left(R^{*}+R\right)^{2} \bar{\tau}_{\bar{X} \bar{R}}\right\} \\
& +\left(\frac{R^{*}}{R^{*}+R}\right) \frac{\partial \bar{\tau}_{\bar{X} \bar{X}}}{\partial X}-\frac{\mu_{e}}{2 \rho} \frac{R^{*}}{R^{*}+\bar{R}}\left(\frac{\partial H^{+^{2}}}{\partial x}\right) \\
& +\frac{\mu_{e}}{\rho}\left[\left(\frac{\bar{h}_{x}}{R^{*}+\bar{R}}+\frac{\partial \bar{h}_{x}}{\partial R}\right)\right. \\
& \left.\left(H_{0} \frac{R^{*}}{R^{*}+\bar{R}}+\bar{h}_{r}\right)+\frac{R^{*}}{R^{*}+\bar{R}} \bar{h}_{\bar{x}} \frac{\partial \bar{h}_{x}}{\partial X}\right], \\
& \bar{\tau}_{\bar{R} \bar{R}}=-\eta_{0}\left[1+\frac{(n-1)}{2} \Gamma^{2}\left\{4\left(\frac{\partial \bar{V}}{\partial \bar{R}}\right)^{2}+\left(\frac{\partial \bar{U}}{\partial \bar{R}}\right.\right.\right. \\
& \left.\left.\left.-\frac{\bar{U}}{\bar{R}+R^{*}}+\frac{R^{*}}{\bar{R}+R^{*}} \frac{\partial \bar{V}}{\partial \bar{X}}\right)^{2}\right\}\right]\left(2 \frac{\partial \bar{V}}{\partial \bar{R}}\right), \\
& \bar{\tau}_{\bar{X} \bar{R}}=-\eta_{0}\left[1+\frac{(n-1)}{2} \Gamma^{2}\left\{4\left(\frac{\partial \bar{V}}{\partial \bar{R}}\right)^{2}\right.\right. \\
& \left.\left.+\left(\frac{\partial \bar{U}}{\partial \bar{R}}-\frac{\bar{U}}{\bar{R}+R^{*}}+\frac{R^{*}}{\bar{R}+R^{*}} \frac{\partial \bar{V}}{\partial \bar{X}}\right)^{2}\right\}\right] \\
& \left(\frac{\partial \bar{U}}{\partial \bar{R}}-\frac{\bar{U}}{\bar{R}+R^{*}}+\frac{R^{*}}{\bar{R}+R^{*}} \frac{\partial \bar{V}}{\partial \bar{X}}\right),
\end{aligned}
$$

$$
\begin{aligned}
\bar{\tau}_{\bar{X} \bar{X}}= & -\eta_{0}\left[1+\frac{(n-1)}{2} \Gamma^{2}\left\{4\left(\frac{\partial \bar{V}}{\partial \bar{R}}\right)^{2}\right.\right. \\
+ & \left.\left.\left(\frac{\partial \bar{U}}{\partial \bar{R}}-\frac{\bar{U}}{\bar{R}+R^{*}}+\frac{R^{*}}{\bar{R}+R^{*}} \frac{\partial \bar{V}}{\partial \bar{X}}\right)^{2}\right\}\right] \\
& \left(-2 \frac{\partial \bar{V}}{\partial \bar{R}}\right) .
\end{aligned}
$$

The transformations between the fixed frame $(\bar{R}, \bar{X})$ and wave frame $(\bar{r}, \bar{x})$ are

$$
\begin{array}{ll}
\bar{x}=\bar{X}-c \bar{t}, & \bar{r}=\bar{R}, \\
\bar{u}=\bar{U}-c, \quad \bar{v}=\bar{V},
\end{array}
$$

and hence Equations. (13)-(18) are reduced to the following expressions

$$
\begin{aligned}
& \frac{\partial \bar{v}}{\partial \bar{r}}+\frac{R^{*}}{\bar{r}+R^{*}} \frac{\partial \bar{u}}{\partial \bar{x}}+\frac{\bar{v}}{r+R^{*}}=0, \\
& \rho\left[-c \frac{\partial \bar{v}}{\partial \bar{x}}+\bar{v} \frac{\partial \bar{v}}{\partial \bar{r}}+\frac{R^{*}(\bar{u}+c)}{R^{*}+\bar{r}} \frac{\partial \bar{v}}{\partial \bar{x}}-\frac{(\bar{u}+c)^{2}}{R^{*}+\bar{r}}\right] \\
= & -\frac{\partial \bar{p}_{m}}{\partial \bar{r}}+\frac{1}{R^{*}+\bar{r}} \frac{\partial}{\partial \bar{r}}\left\{\left(R^{*}+R\right) \bar{\tau}_{r r}\right\} \\
& +\left(\frac{R^{*}}{R^{*}+\bar{r}}\right) \frac{\partial \bar{\tau}_{x r}}{\partial \bar{x}}-\frac{\bar{\tau}_{x x}}{\bar{r}+R^{*}}-\frac{\mu_{e}}{2}\left(\frac{\partial H^{+^{2}}}{\partial \bar{r}}\right) \\
& +\mu_{e}\left[\left(H_{0} \frac{R^{*}}{R^{*}+\bar{r}}+\bar{h}_{\bar{r}}\right) \frac{\partial \bar{h}_{\bar{r}}}{\partial \bar{r}}\right. \\
& \left.+\bar{h}_{\bar{x}} \frac{R^{*}}{R^{*}+\bar{r}} \frac{\partial \bar{h}_{\bar{r}}}{\partial \bar{x}}-\frac{\bar{h}_{\bar{x}}^{2}}{R^{*}+\bar{r}}\right], \\
& \rho\left[-c \frac{\partial u}{\partial \bar{x}}+\bar{v} \frac{\partial \bar{u}}{\partial \bar{r}}+\frac{R^{*}(\bar{u}+c)}{R^{*}+\bar{r}} \frac{\partial \bar{u}}{\partial \bar{x}}-\frac{(\bar{u}+c) \bar{v}}{R^{*}+\bar{r}}\right] \\
= & -\frac{R^{*}}{R^{*}+\bar{r}} \frac{\partial \bar{p}_{m}}{\partial \bar{x}}+\frac{1}{\left(R^{*}+\bar{r}\right)^{2}} \frac{\partial}{\partial \bar{r}}\left\{\left(R^{*}+\bar{r}\right)^{2} \bar{\tau}_{x r}\right\} \\
& +\left(\frac{R^{*}}{R^{*}+\bar{r}}\right) \frac{\partial \bar{\tau}_{x x}}{\partial \bar{x}}-\frac{\mu_{e}}{2} \frac{R^{*}}{R^{*}+\bar{r}}\left(\frac{\partial H^{++^{2}}}{\partial x}\right) \\
& +\mu_{e}\left[\left(\frac{\bar{h} \bar{x}}{R^{*}+\bar{r}}+\frac{\partial \bar{h}_{\bar{x}}}{\partial \bar{r}}\right)\right. \\
& \left.\left(H_{0} \frac{R^{*}}{R^{*}+\bar{r}}+\bar{h}_{\bar{r}}\right)+\frac{R^{*}}{R^{*}+\bar{r}} \bar{h}_{\bar{x}} \frac{\partial \bar{h}_{\bar{x}}}{\partial \bar{x}}\right], \quad(22)
\end{aligned}
$$




$$
\begin{aligned}
\bar{\tau}_{r r}= & -\eta_{0}\left[1+\frac{(n-1)}{2} \Gamma^{2}\left\{4\left(\frac{\partial v}{\partial r}\right)^{2}\right.\right. \\
+ & \left.\left.\left(\frac{\partial u}{\partial r}-\frac{(u+c)}{r+R^{*}}+\frac{R^{*}}{r+R^{*}} \frac{\partial v}{\partial x}\right)^{2}\right\}\right] \\
& \left(2 \frac{\partial v}{\partial r}\right)
\end{aligned}
$$$$
\bar{\tau}_{r x}=-\eta_{0}\left[1+\frac{(n-1)}{2} \Gamma^{2}\left\{4\left(\frac{\partial v}{\partial r}\right)^{2}\right.\right.
$$$$
\left.\left.+\left(\frac{\partial u}{\partial r}-\frac{(u+c)}{r+R^{*}}+\frac{R^{*}}{r+R^{*}} \frac{\partial v}{\partial x}\right)^{2}\right\}\right]
$$$$
\left(\frac{\partial u}{\partial r}-\frac{(u+c)}{r+R^{*}}+\frac{R^{*}}{r+R^{*}} \frac{\partial v}{\partial x}\right),
$$$$
\bar{\tau}_{x x}=-\eta_{0}\left[1+\frac{(n-1)}{2} \Gamma^{2}\left\{4\left(\frac{\partial v}{\partial r}\right)^{2}\right.\right.
$$$$
\left.\left.+\left(\frac{\partial u}{\partial r}-\frac{(u+c)}{r+R^{*}}+\frac{R^{*}}{r+R^{*}} \frac{\partial v}{\partial x}\right)^{2}\right\}\right]
$$$$
\left(-2 \frac{\partial v}{\partial r}\right)
$$

We now define

$$
\begin{aligned}
x & =\frac{\bar{x}}{\lambda}, \quad r=\frac{\bar{r}}{a}, \quad t=\frac{c \bar{t}}{\lambda}, \\
p & =\frac{a^{2} \bar{p}}{c \lambda \eta_{0}}, \quad M^{2}=\operatorname{ReS}^{2} R_{m}, \quad \delta=\frac{a}{\lambda} \\
W e & =\frac{\Gamma c}{a}, \quad \tau_{i j}=\frac{a \bar{\tau}_{i j}}{c \eta_{0}}, \quad u=\frac{\bar{u}}{c}, \\
v & =\frac{\bar{v}}{c}, \quad k=\frac{R^{*}}{a}, \quad E=\frac{-\bar{E}}{c H_{0} \mu_{e}}, \\
\operatorname{Re} & =\frac{c a \rho}{\eta_{0}}, \quad R_{m}=\sigma \mu_{e} a c, \\
S & =\frac{H_{0}}{c} \sqrt{\frac{\mu_{e}}{\rho}}, \quad \phi=\frac{\bar{\phi}}{H_{0} a}, \quad u=-\frac{\partial \psi}{\partial r}, \\
\bar{h}_{\bar{x}} & =-\bar{\phi}_{\bar{r}}, \quad \bar{h}_{\bar{r}}=\frac{R^{*}}{R^{*}+\bar{r}} \bar{\phi}_{\bar{x}}, \\
p_{m} & =p+\frac{1}{2} \operatorname{Re} \frac{\mu_{e}\left(H^{+}\right)^{2}}{\rho c^{2}}, h_{x}=-\frac{\partial \phi}{\partial r},
\end{aligned}
$$

$$
v=\delta \frac{k}{k+r} \frac{\partial \psi}{\partial x}, h_{r}=\delta \frac{k}{k+r} \frac{\partial \phi}{\partial x},
$$

where $\psi$ is the stream function, $\phi$ is the magnetic force function, $p_{m}$ the total pressure (sum of ordinary and magnetic pressures), $E$ the electric field strength and $\delta, W e, R e, R_{m}, S$ and $M$ are the wave, Weissenberg, Reynolds, magnetic Reynolds, Stommer's and Hartman numbers respectively. Equation (20) is satisfied identically in view of Equation (26) and other Equations yield

$$
\begin{aligned}
& \frac{\operatorname{Re} \delta}{k+r}\left[-\delta^{2} k \frac{\partial^{2} \psi}{\partial x^{2}}+\frac{\delta^{2} k^{2}}{k+r}\left\{\frac{\partial \psi}{\partial x} \frac{\partial^{2} \psi}{\partial r \partial x}\right.\right. \\
& \left.\left.+\left(1-\frac{\partial \psi}{\partial r}\right) \frac{\partial^{2} \psi}{\partial x^{2}}\right\}-\left(1-\frac{\partial \psi}{\partial r}\right)^{2}\right] \\
& =-\frac{\partial p_{m}}{\partial r}-\frac{\delta}{k+r} \\
& \left.+\frac{\partial}{\partial r}(r+k) \tau_{r r}-\delta k \frac{\partial}{\partial x} \tau_{r x}-\frac{\tau_{x x}}{r+k}\right] \\
& +\frac{\operatorname{Re} \delta S^{2}}{k+r}\left[\frac{k^{2} \delta}{k+r}\left(1+\delta \frac{\partial \phi}{\partial x}\right) \frac{\partial^{2} \phi}{\partial r \partial x}\right. \\
& \left.-\frac{k^{2} \delta^{2}}{k+r} \frac{\partial \phi}{\partial r} \frac{\partial^{2} \phi}{\partial x^{2}}-\frac{\partial^{2} \phi}{\partial r^{2}}\right],
\end{aligned}
$$

$$
\begin{aligned}
& \frac{\operatorname{Re} \delta(k+r)}{k}\left[\frac{\partial^{2} \psi}{\partial x \partial r}+\frac{k}{k+r}\left\{\frac{\partial \psi}{\partial x} \frac{\partial^{2} \psi}{\partial r \partial x}\right.\right. \\
& \left.+\left(1-\frac{\partial \psi}{\partial r}\right) \frac{\partial^{2} \psi}{\partial x \partial r}\right\} \\
& \left.-\frac{k}{(r+k)^{2}}\left(1-\frac{\partial \psi}{\partial r}\right) \frac{\partial \psi}{\partial x}\right] \\
& =-\frac{\partial p_{m}}{\partial x}+\frac{1}{k(r+k)} \frac{\partial}{\partial r}\left((r+k)^{2} \tau_{r x}\right)-\delta \frac{\partial}{\partial x} \tau_{x x} \\
& +\frac{\operatorname{Re}(k+r) S^{2}}{k}\left[\frac{k}{k+r}\left(1+\delta \frac{\partial \phi}{\partial x}\right)\right. \\
& \left.\left(\frac{1}{k+r} \frac{\partial \phi}{\partial r}+\frac{\partial^{2} \phi}{\partial r^{2}}\right)-\frac{k \delta}{k+r} \frac{\partial \phi}{\partial r} \frac{\partial^{2} \phi}{\partial x \partial r}\right], \\
& E=\frac{k}{k+r} \frac{\partial \psi}{\partial r}-\delta \frac{k}{k+r} \frac{\partial \psi}{\partial x} \frac{\partial \phi}{\partial r}+\frac{1}{R_{m}} \nabla^{2} \phi,(30) \\
& \nabla^{2}=\left[\frac{\partial^{2}}{\partial r^{2}}+\frac{1}{k+r} \frac{\partial}{\partial r}+\delta^{2} \frac{k^{2}}{(k+r)^{2}} \frac{\partial^{2}}{\partial r^{2}}\right] .
\end{aligned}
$$


Adopting long wavelength and low Reynolds number considerations we arrive at

$$
\begin{gathered}
\frac{\partial p}{\partial x}=-\frac{1}{k(k+r)} \frac{\partial}{\partial r}\left[(k+r)^{2} \tau_{r x}\right] \\
+\operatorname{Re} S^{2}\left[\frac{1}{k+r} \frac{\partial \phi}{\partial r}+\frac{\partial^{2} \phi}{\partial r^{2}}\right], \\
E=\frac{k}{k+r} \frac{\partial \psi}{\partial r}+\frac{1}{R_{m}}\left[\frac{\partial^{2} \phi}{\partial r^{2}}+\frac{1}{k+r} \frac{\partial \phi}{\partial r}\right], \\
\tau_{r x}=-\left[1+\frac{(n-1)}{2} W e^{2}\right. \\
\left.\left\{\psi_{r r}-\frac{1}{k+r}\left(1-\psi_{r}\right)\right\}^{2}\right] \\
\left(-\psi_{r x}+\frac{1}{k+r}\left(1-\psi_{r}\right)\right)
\end{gathered}
$$

where Equation (28) results in that $p \neq p(r)$. The subjected conditions are

$$
\begin{aligned}
& \Psi=-\frac{F}{2}, \quad \frac{\partial \Psi}{\partial r}=1, \quad \phi=0 \text { at } \quad y=h, \\
& \Psi=\frac{F}{2}, \quad \frac{\partial \Psi}{\partial r}=1, \quad \phi=0 \text { at } \quad y=-h,
\end{aligned}
$$

where the dimensionless time mean flow rate $F$ in the wave frame is related to the dimensionless time mean flow rate $\theta$ in the laboratory frame by the following relations

$$
\theta=F+2, \quad F=-\int_{-h}^{h} \frac{\partial \Psi}{\partial r} d r .
$$

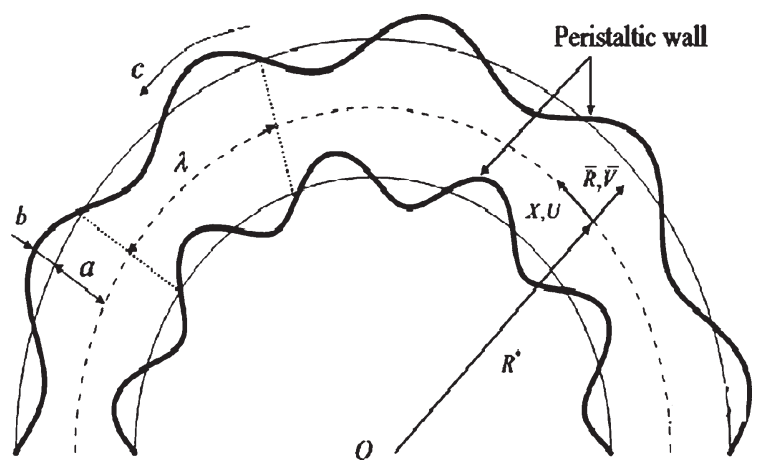

Fig. 1. Geometry of flow problem.

\section{Solution expressions}

Expanding $\psi, F, p, \phi$ and $\tau_{r x}$ in terms of perturbation quantity $W e^{2}$ and using in Equations. (30-34) and then solving the resulting zeroth and first order systems and employing

$$
F_{0}=F-W e^{2} F_{1},
$$

we finally obtain

$$
\begin{aligned}
\psi(r)= & C_{1}-\frac{k+r}{b^{2}}+C_{2}(k+r)^{2} \\
& +(r+k)\left(C_{3} \cos (b \ln (r+k))\right. \\
& \left.+C_{4} \sin (b \ln (r+k))\right) \\
& +W e^{2}\left[A_{1}+A_{2}(r+k)^{2}\right. \\
& +(r+k)\left(A_{3} \cos (b \ln (r+k))\right. \\
& \left.+A_{4} \sin (b \ln (r+k))\right)-\frac{1}{2(r+k)} \frac{(n-1)}{2} \\
& \left\{L_{0}+L_{1} \ln (r+k) \sin (b \ln (r+k))\right. \\
+ & \cos (b \ln (r+k)) \\
+ & L_{3} \times \cos (3 b \ln (r+k)) L_{2} \ln (r+k) \\
+ & L_{4} \sin (3 b \ln (r+k))+\cos (2 b \ln (r+k)) \\
& \left.\left.+L_{6} \sin (2 b \ln (r+k)) L_{5}\right\}\right],
\end{aligned}
$$

$$
\begin{aligned}
\phi(r)= & B_{1}+B_{2} \ln ((r+k) \\
& -\frac{1}{4} R_{m} W e^{2} L_{0}(\ln (r+k))^{2} \\
& +\frac{1}{4} R_{m}\left(E+\frac{1+W e^{2}}{b^{2}}\right) L_{0}(r+k)^{2} \\
& -\frac{2 R_{m}}{4 b^{2}}\left(C_{2}+W e^{2} A_{2}\right)(r+k)^{3} \\
& -\frac{L_{1} W e^{2} R_{m}}{b^{2}} \sin (b \ln (r+k)) \\
& -\cos (b \ln (r+k)) \times \frac{L_{1} W e^{2} R_{m}}{b^{2}} \\
& +\frac{W e^{2} R_{m}}{4 b^{2}}\left(2 b L_{5}+L_{6}\right) \sin (2 b \ln (r+k)) \\
& +\frac{W e^{2} R_{m}}{4 b^{2}}\left(-2 b L_{6}+L_{5}\right) \\
& \times \cos (2 b \ln (r+k))
\end{aligned}
$$




$$
\begin{aligned}
+ & \frac{W e^{2} R_{m}}{9 b^{2}}\left(3 b L_{3}+L_{4}\right) \sin (3 b \ln (r+k)) \\
+ & \frac{W e^{2} R_{m}}{9 b^{2}}\left(-3 b L_{3}+L_{4}\right) \cos (3 b \ln (r+k)) \\
+ & \frac{W e^{2} R_{m}}{b^{2}}\left(b L_{1}-L_{2}\right)(-\ln ((r+k) \\
& \left.\cos (3 b \ln (r+k))+\frac{2}{b} \sin (b \ln (r+k))\right) \\
+ & (\ln (r+k) \sin (b \ln (r+k)) \\
+ & \left.\frac{2}{b} \cos (b \ln (r+k))\right)\left(L_{1}+b L_{2}\right) \\
- & \frac{R_{m}(r+k)^{2}}{b^{4}+8 b^{2}+16}\left(\left(4-b^{2}\right) \cos (b \ln (r+k))\right. \\
+ & \left.\frac{4}{b} \sin (b \ln (r+k))\right) \\
& \left(C_{3}+b C_{4}+W e^{2}\left(A_{3}+b A_{4}\right)\right)
\end{aligned}
$$

$$
\begin{aligned}
- & \frac{R_{m}(r+k)^{2}}{b^{4}+8 b^{2}+16}\left(C_{4}-b C_{3}\right. \\
+ & \left.W e^{2}\left(A_{4}-b A_{3}\right)\right) \\
& \left(\left(4-b^{2}\right) \sin (b \ln (r+k))\right. \\
- & \left.\frac{4}{b} \cos (b \ln (r+k))\right),
\end{aligned}
$$$$
b=\sqrt{k^{2} M^{2}-1}
$$

$$
\begin{aligned}
\frac{d p}{d x}= & -\frac{1}{k(k+r)} \frac{\partial}{\partial r}\left[( k + r ) ^ { 2 } \left(\frac{\partial^{2} \psi}{\partial r^{2}}\right.\right. \\
& +\frac{1}{k+r}\left(1-\frac{\partial \psi}{\partial r}\right)+\frac{(n-1)}{2}\left\{\frac{\partial^{2} \psi_{0}}{\partial r^{2}}\right. \\
& \left.\left.+\frac{1}{k+r}\left(1-\frac{\partial \psi_{0}}{\partial r}\right)\right\}^{3}\right] \\
& +M^{2}\left[E-\frac{k}{k+r} \frac{\partial \psi}{\partial r}\right],
\end{aligned}
$$
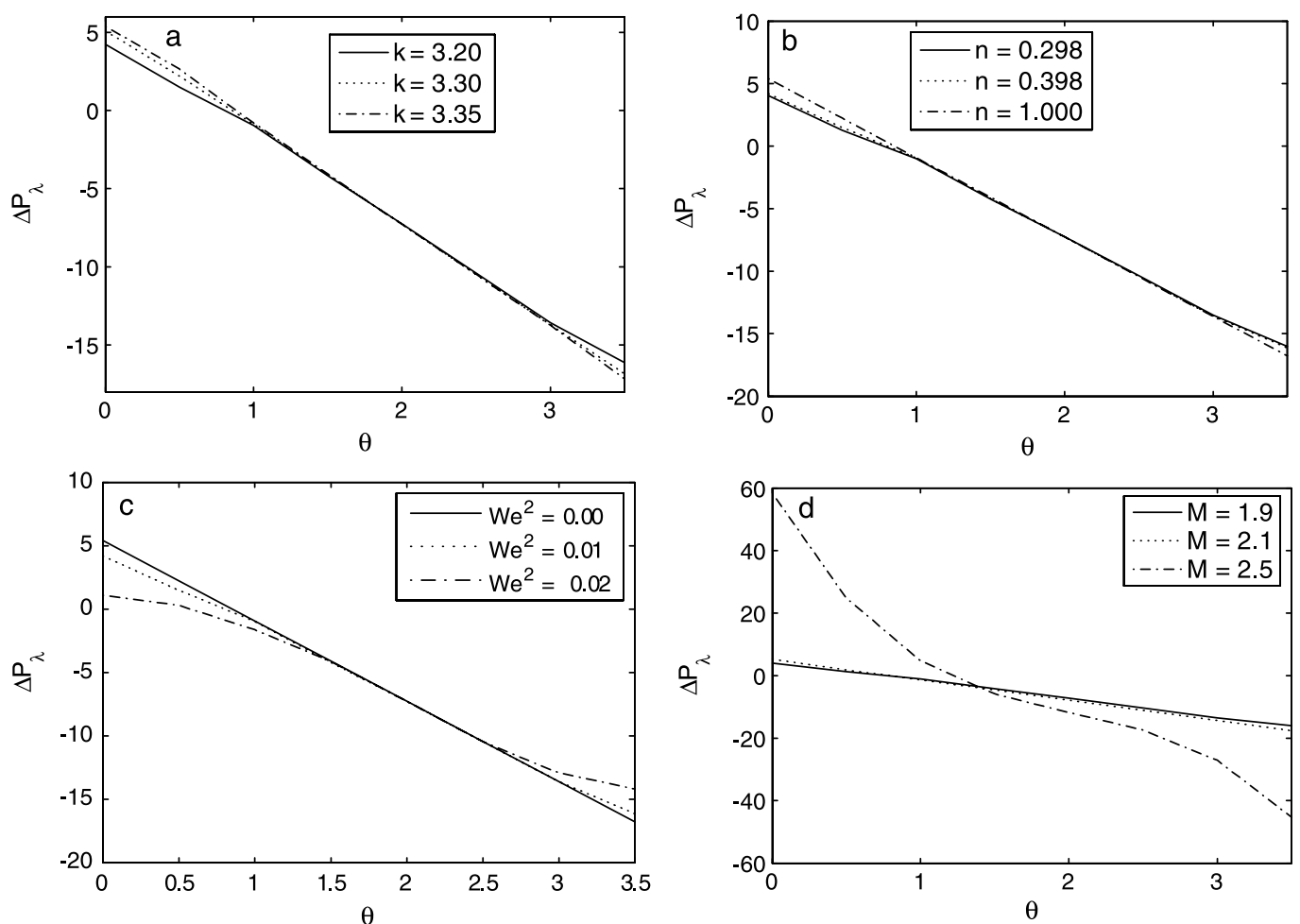

Fig. 2. (a) The pressure rise $\Delta P_{\lambda}$ versus flow rate $\theta$ for $E=0, n=0.398, \alpha=0.4, W e=0.002$ and $M=1.9$. (b) The pressure rise $\Delta P_{\lambda}$ versus flow rate $\theta$ for $E=0, k=3.2, \alpha=0.6, W e=0.002, M=1.9$ and $k=3.2$. (c) The pressure rise $\Delta P_{\lambda}$ versus flow rate $\theta$ for $E=0, k=3.2, \alpha=$ $0.6, n=0.398, M=1.9$ and $k=3.2$. (d) The pressure rise $\Delta P_{\lambda}$ versus flow rate $\theta$ for $E=0, k=3.2, \alpha=0.6, n=0.298, W e=0.002$ and $k=3.2$. 
and the involved $C_{i}(i=1-4), A_{i}(i=1-4)$, $B_{i}(i=1,2), L_{i}(i=1-6)$ and $l_{i}(i=1-7)$ can be computed algebraically. Axial induced magnetic field $h_{x}$, current density $J_{z}$ and pressure rise $\Delta P_{\lambda}$ can be computed by following relations

$$
\begin{aligned}
h_{x} & =-\frac{\partial \phi}{\partial r}, \quad J_{z}=-\frac{\partial h_{x}}{\partial r}, \\
\Delta P_{\lambda} & =\int_{0}^{1}\left(\frac{d p}{d x}\right)_{r=0} d x .
\end{aligned}
$$

\section{Results and discussion}

This section explores the influence of $M, k, n$ and $R_{m}$ on axial velocity $u$, pressure rise per wavelength $\Delta P_{\lambda}$, axial induced magnetic field $h_{x}$ and current density $J_{Z}$. Theoretical results are presented for both curved and straight geometries. It is found that zigzag channel outperforms the straight channel.
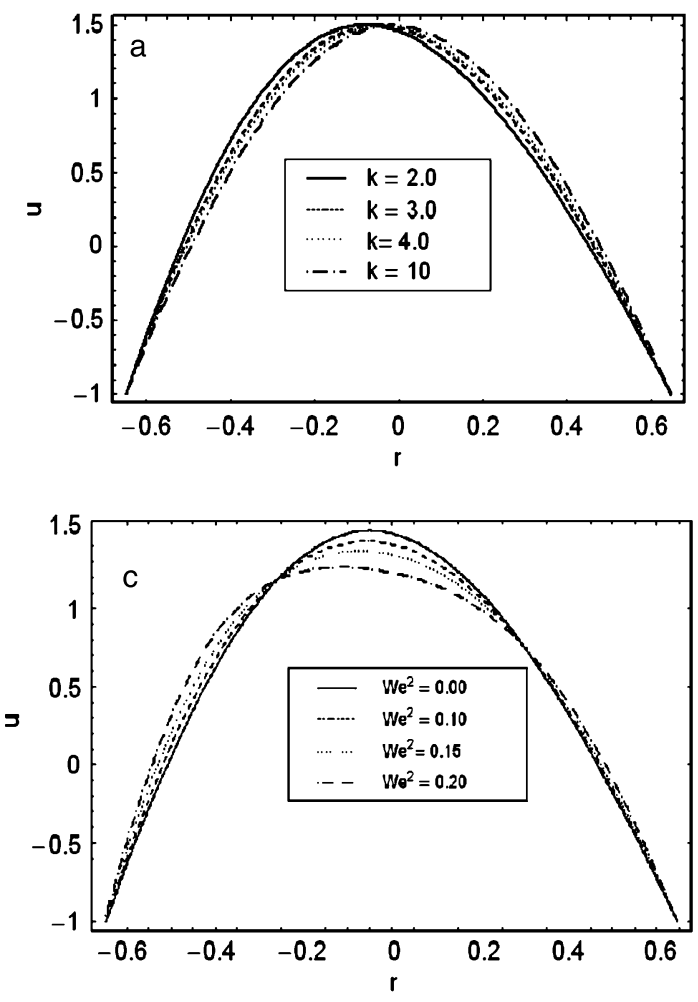

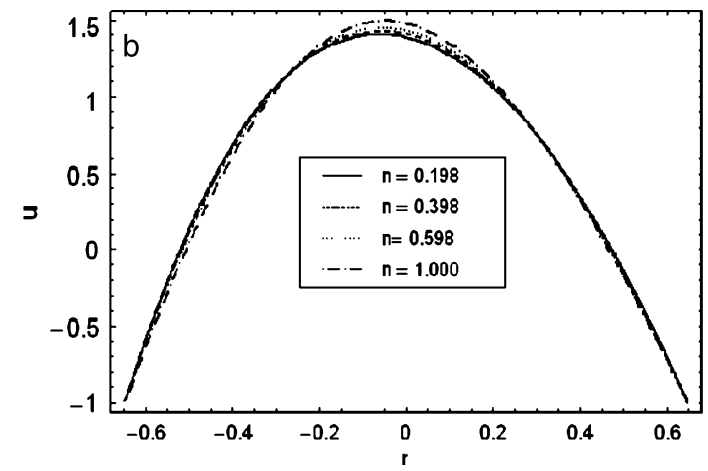

\subsection{Pumping characteristics}

Figure 2a plots the effect of curvature parameter $k$ on pressure rise. It has been noticed that the presence of curvature increases $\Delta P_{\lambda}$ in the pumping region [26]. It is further noted that there is an increase in pressure rise when one moves from curved to straight channel i.e., peristalsis has to work against lesser pressure rise in curved channel than in straight channel. There is also an increase in the free pumping flux when one moves from curved to straight channel. In copumping region, where the pressure assists the flow, mixed behavior of curvature parameter is observed for the fixed values of flow rate.

Figure $2 b$ discusses the variation of $n$ on pressure rise per wavelength. It is observed that pumping curves for Carreau fluid lie below the curves for Newtonian fluid and these curves tend to coincide with the curves for Newtonian fluid as $n$ increases. Moreover, for some fixed values of flow rate there is no difference between the Newtonian and Carreau fluids. In Fig. 2c we noticed

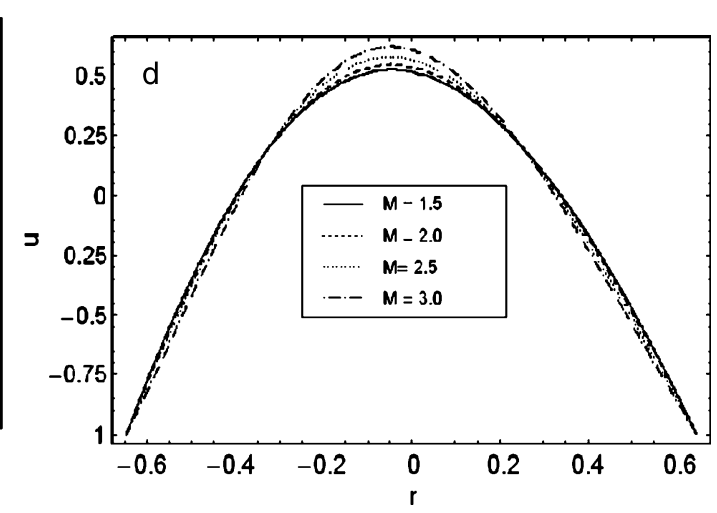

Fig. 3. (a) The axial velocity $u$ versus $r$ for $\alpha=0.6, W e=0.02, \theta=2.8, x=0.6, M=1.9$ and $n=0.398$. (b) The axial velocity $u$ versus $r$ for $\alpha=0.6, W e=0.1, \theta=2.8, x=0.6, M=1.9$ and $k=3.2$. (c) The axial velocity $u$ versus $r$ for $\alpha=0.6, n=0.398, \theta=2.8, x=0.6, M=1.9$ and $k=3.2$. (d) The axial velocity $u$ versus $r$ for $\alpha=0.6, n=0.298, \theta=2, x=0.6, W e=0.02$ and $k=3.2$. 

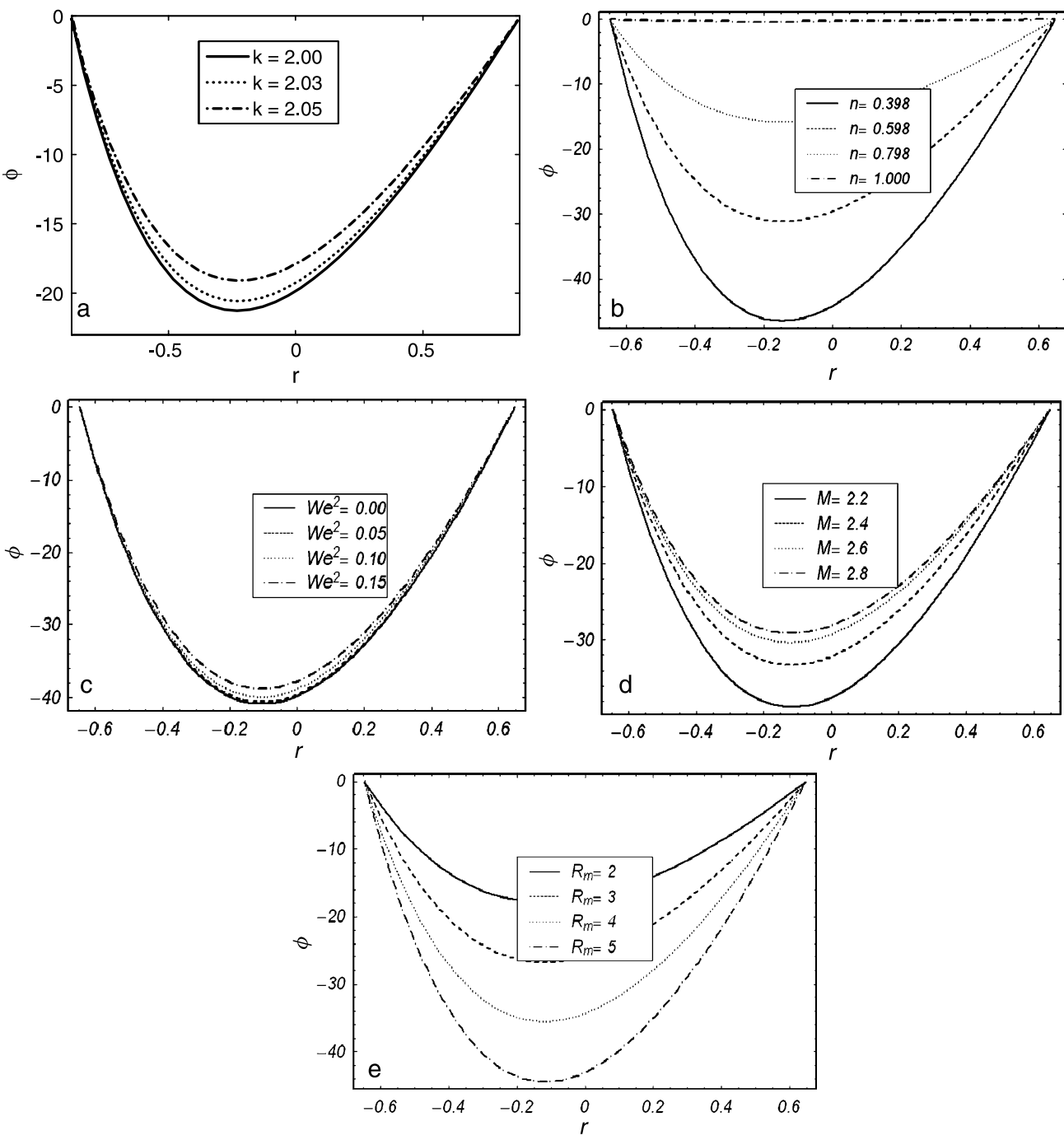

Fig. 4. (a) The magnetic force function $\phi$ versus $r$ for $n=0.398, \alpha=0.2, \theta=-2, E=1, x=0.6, W e=0.02, R_{m}=4$ and $M=2$. (b) The magnetic force function $\phi$ versus $r$ for $k=1.5, \alpha=0.6, \theta=-2.8, E=1, x=0.6, W e=0.02, R_{m}=2$ and $M=1.9$. (c) The magnetic force function $\phi$ versus $r$ for $k=2.2, \alpha=0.6, \theta=-2.8, E=1, x=0.6, n=0.398, R_{m}=4$ and $M=2$. (d) The magnetic force function $\phi$ versus $r$ for $k=2.2, \alpha=0.6, \theta=-2.8, E=1, x=0.6, n=0.398, R_{m}=4$ and $M=2$. (e)The magnetic force function $\phi$ versus $r$ for $k=2$, $\alpha=0.6, \theta=-2.8, E=1, x=0.6, n=0.398, W e=0.02$ and $M=2.3$

that in pumping region $\left(\Delta p_{\lambda}>0, \theta>0\right)$, an increase in We decreases the pumping rate. For the case of copumping there is no difference between the Newtonian and Carreau fluids.

The variation of pressure rise $\Delta p_{\lambda}$ against mean flow rate $\theta$ for different values of $M$ is depicted in Fig. 2d. Here an increase in $M$ causes an increase in the pressure rise. It is found that $\Delta P_{\lambda}$ in the pumping region $\left(\Delta p_{\lambda}>0, \theta>0\right)$ increases by increasing $M$ for the fixed values of flow rate. However for the case of copumping $\left(\Delta p_{\lambda}<0\right)$ the flow rate $\theta$ decreases when $M$ is increased.

\subsection{Flow characteristics}

A flow proceeding up a curved channel is the same flow, even though its direction varies continuously along the channel. Curved channels provide greater length for the same disc diameter compared to straight. The axial velocity $u$ for various values of curvature 

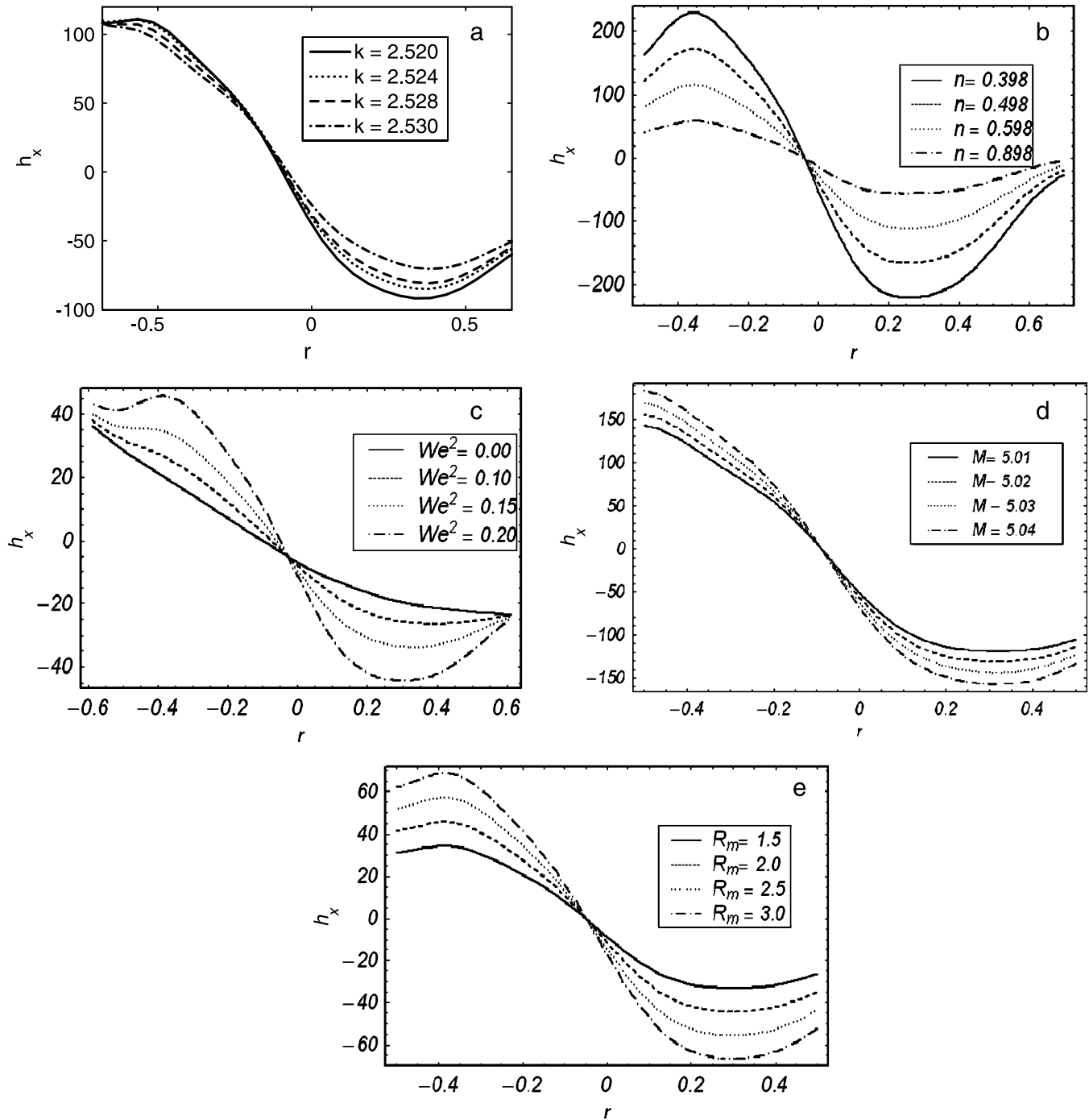

Fig. 5. (a) The axial induced magnetic field $h_{x}$ versus $r$ for $\alpha=0.2, \theta=5, E=-3, x=-0.2, n=0.898, W e=0.3, R_{m}=4$ and $M=5$. (b) The axial induced magnetic field $h_{x}$ versus $r$ for $\alpha=0.3, \theta=-0.2, E=-1, x=-0.2, k=2.5$, We $=0.5, R_{m}=2$ and $M=6.25$. (c) The axial induced magnetic field $h_{x}$ versus $r$ for $\alpha=0.3, \theta=-0.6, E=-1, x=-0.2, k=2.5, n=0.9, R_{m}=2$ and $M=6.25$. (d) The axial induced magnetic field $h_{x}$ versus $r$ for $\alpha=0.2, \theta=5, E=-3, x=-0.2, k=2.5, n=0.898, R_{m}=4$ and $W e=0.3$. (e)The axial induced magnetic field $h_{x}$ versus $r$ for $\alpha=0.3, \theta=-0.6, E=-1, x=-0.2, k=2.5, n=0.9, M=6.25$ and $W e=0.2$.

parameter $k$ is sketched in Fig. 3a. We observed that profiles are not symmetric about $r=0$. There is a shift of profiles for smaller values of $k$ (i.e., an increase in the curvature of channel) towards the lower wall. However, symmetry is possible when $k \rightarrow \infty$.

Figure $3 b$ shows that axial velocity increases when the power law index increases for $r=0$. Figure $3 \mathrm{c}$ depicts that maxima in $u(r)$ for Carreau fluid $W e(\neq 0)$ lies below the maxima of Newtonian fluid $(W e=0)$. Hence the velocity in Carreau fluid is smaller than Newtonian fluid.
The variation of Hartman number $M$ on $u$ is shown in Fig. 3d. We notice that velocity profile is not symmetric about the central line of the channel due to curvature. Behavior of $M$ near the walls of channel is quite opposite to that at the centre of channel. At $r=0$ magnitude of velocity is an increasing function of $M$.

\subsection{Magnetic field characteristics}

This discussion yields insights into the mechanisms of blood flow in curved channels having frictionless 

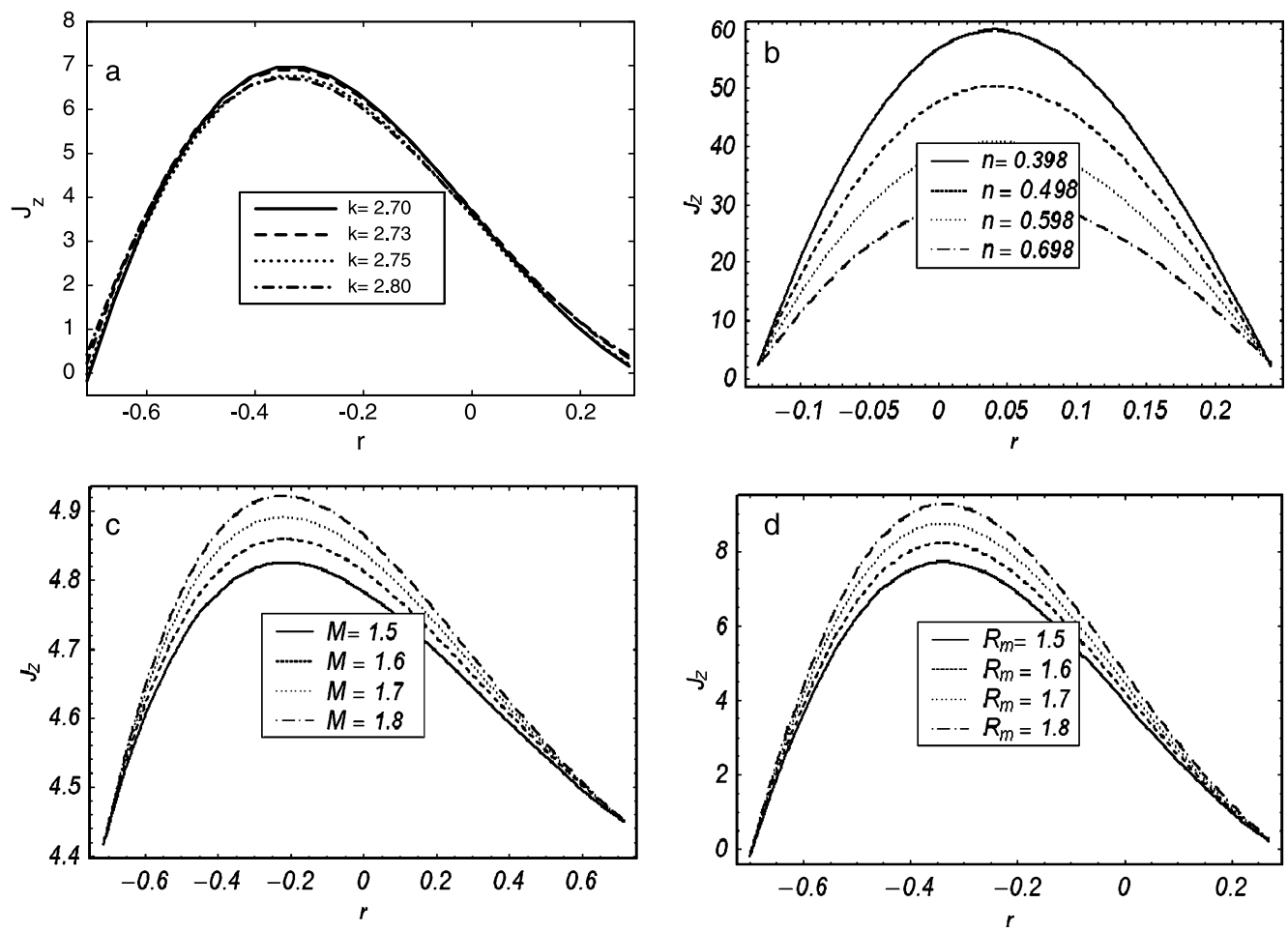

Fig. 6. (a) Current density versus $J_{z}$ versus $r$ for $\theta=0.5, \alpha=0.3, R_{m}=1.5, E=-1, x=-0.2, n=0.9, M=3.3$ and $W e=0.9$. (b) Current density $J_{z}$ versus $r$ for $\alpha=0.3, \theta=0.5, R_{m}=1.5, E=-1, x=-0.2, k=2.5, M=6.23$ and $W e=0.6$. (c) Current density $J_{z}$ versus $r$ for $\alpha=0.3, \theta=0.5, R_{m}=2, E=-1, x=-0.2, k=2.5, n=0.9$ and $W e=0.9$. (d) Current density $J_{z}$ versus $r$ for $\alpha=0.3, \theta=0.5$, $M=3.3, E=-1, x=-0.2, k=2.5, n=0.9$ and $W e=0.9$.

walls exposed to a magnetic field. Blood is a biomagnetic fluid. Figure $4 \mathrm{a}-\mathrm{e}$ explain the magnetic field characteristics $k, n, W e$ and $M$. One observes that the parabolic profiles for magnetic force function depict a left shift at $r=0$ due to curvature effects. Magnetic force function is zero at the walls, which is in complete agreement with the imposed boundary condition. The profiles here are increasing function of $k, n$, We and $M$ near the upper wall of channel. Magnetic force function is decreasing function of $R_{m}$ (Fig. 4e).

Figure 5a-e discuss the axial induced magnetic field $h_{x}$ against $r$ for different values of $k, n, W e, M$ and $R_{m}$. In the half region induced magnetic field is in one direction whereas in the other half it is in opposite direction. Magnitude of $h_{x}$ increases with $W e, M$ and $R_{m}$ while shows an opposite behavior for $k$ and $n$.

The current density distribution $J_{z}$ for various values of $k, n, M$ and $R_{m}$ is plotted in the Fig. 6a-d. Here the magnitude of current density $J_{z}$ decreases about the centre of channel by increasing $k$ and $n$. A shift in the profiles is observed towards the lower wall. For large values of $k$ symmetry in the profiles is obtained and results of planar channel are deduced. Both $M$ and $R_{m}$ have increasing effects on the current density distribution (Fig. 6c and d).

\section{Concluding remarks}

We have discussed the flow characteristics of Carreau fluid in a curved channel. The main observations are as follows.

- Axial velocity for Carreau fluid is smaller than viscous fluid about the centre of channel.

- Pressure rise per wavelength and axial velocity are greater for viscous fluid in comparison to Carreau fluid.

- The tilt in velocity profiles shows that flow is more towards the lower wall.

- Magnitude of induced magnetic field is greater for Carreau fluid in comparison to viscous fluid. 
- Symmetry of quantities of interest such as velocity, current density and induced magnetic filed at $r=0$ is disturbed due to curvature. The results of planar channel are recovered when $k \rightarrow \infty$.

\section{Acknowledgment}

The research of Dr. Alsaedi was partially supported by Deanship of Scientific Research (DSR), King Abdulaziz University, Jeddah, Saudi Arabia.

\section{References}

[1] T.W. Latham, Fluid motion in a peristaltic pump, MS Thesis, MIT Cambridge MA, 1966.

[2] A.H. Shapiro, M.Y. Jaffrin and S.L. Weinberg, Peristaltic pumping with long wavelength at low Reynolds, J Fluid Mech 37 (1969), 799-825.

[3] N. Ali, T. Hayat and S. Asghar, Peristaltic flow of a Maxwell fluid in a channel with compliant walls, Chaos, Solitons \& Fractals 39 (2009), 407-416.

[4] S. Srinivas and M. Kothandapani, Peristaltic transport in an asymmetric channel with heat transfer - A note, Int Commun Heat Mass Transf 35 (2008), 514-522.

[5] M. Kothandapani and S. Srinivas, Non-linear peristaltic transport of a Newtonian fluid in an inclined asymmetric channel through a porous medium, Physics Lett A 372 (2008), $1265-1276$

[6] M.V.S. Reddy, A.R. Rao and S. Sreenadh, Peristaltic motion of a power-law fluid in an asymmetric channel, Int J Nonlin Mech 42 (2007), 1153-1161.

[7] S. Nadeem and S. Akram, Peristaltic flow of a Williamson fluid in an asymmetric channel, Comm Nonlinear Sci Numer Simul 15 (2010), 1705-1716.

[8] D. Tripathi, Peristaltic flow of a fractional second grade fluid through acylindrical tube, Thermal Sci, Doi:10.2298/ TSCI100503061T

[9] D. Tripathi, S.K. Pandey, S.Das, Peristaltic flow of viscoelastic fluid with fractional maxwell model through a channel, Appl Math Comp 215 (2010), 3645-3654.

[10] T. Hayat, S. Noreen, N. Ali and S. Abbasbanday, Peristaltic motion of Phan-Thien-Tanner fluid in a planar channel, Num Meth Part Diff Eqs, Doi 10.1002/num.20647.

[11] P.H. Robert, An intoduction to magnetohydodynamicss, The Whitefriars Press Ltd, London, 1967.
[12] E.E. Tzirtzilakis, A mathematical model for blood flow in magnetic field, Phys Fluids 17 (2005), 077-103.

[13] Abd El Hakeem Abd El Naby, A.E.M. El Misery and M.F. Abd El Kareem, Effects of magnetic field on trapping through peristaltic motion for generalized Newtonian fluid in channel, Physica A 367 (2006), 79-92.

[14] N.T.M. Eldabe, M.F. Al-Sayad, A.Y. Galy and H.M. Sayed, Peristaltically induced motion of a MHD biviscosity fluid in a non-uniform tube, Physica A $\mathbf{3 8 3}$ (2007), 253-266.

[15] S. Srinivas and R. Muthuraj, Effects of chemical reaction and space porosity on MHD mixed convective flow in a vertical asymmetric channel with peristalsis, Math Comp Mod $\mathbf{5 4}$ (2011), 1213-1227.

[16] M. Kothandapani and S. Srinivas, Peristaltic transport of a Jeffrey fluid under the effect of magnetic field in an asymmetric channel, Int J Non-Linear Mech 43 (2008), 915.

[17] T. Hayat and N. Ali, Peristaltic motion of a Jeffrey fluid under the effect of a magnetic field in a tube, Comm Nonlinear $\mathrm{Sci}$ Numer Simul 13 (2008), 1343-1352.

[18] V.I. Vishnyakov and K.B. Pavlov, Peristaltic flow of a conductive fluid in a transverse magnetic field, Translated from Magnitnaya Gidrodinamika 8 (1972), 174-178.

[19] Kh.S. Mekheimer, Effect of induced magnetic field on peristaltic flow of a couple stress fluid, Phys Lett A 372 (2008), 4271-4278.

[20] Kh.S. Mekheimer, Peristaltic flow of a magneto-micropolar fluid: Effect of induced magnetic field, J Appl Math 2008 (2008), 570825-570848.

[21] T. Hayat, Y. Khan, N. Ali and Kh. S. Mekheimer, Effect of an induced magnetic field on the peristaltic flow of a third order fluid, Numer Methods Partial Diff Eqs 26 (2010), 345-360.

[22] T. Hayat, N. Saleem and N. Ali, Effect of induced magnetic field on peristaltic transport of a Carreau fluid, Comm Nonlinear Sci Numer Simul 15 (2010), 2407-2423.

[23] T. Hayat and S. Noreen, Peristaltic transport of fourth grade fluid with heat transfer and induced magnetic field, $C . R$ Mécanique 338 (2010), 518-528.

[24] T. Hayat, S. Noreen, S.Asghar and A. Awatif, Hendi, Influence of induced magnetic field on the peristaltic transport in an asymmetric channel, Chem Engg Comm 198 (2011), 609-628.

[25] T. Hayat, S. Noreen and N. Ali, Effect of an induced magnetic field on the peristaltic motion of Phan-Thien-Tanner (PTT) fluid, Z Naturforsch 65a (2010), 665-676.

[26] N. Ali, M. Sajid and T. Hayat, Long wavelength flow analysis in a curved channel, Zeitschrift Fur Naturforschung A $\mathbf{6 5}$ (2010), 191-196.

[27] N. Ali, M. Sajid, T. Javed and Z. Abbas, Heat transfer analysis of peristaltic flow in a curved channel, Int J Heat Mass Transfer 53 (2010), 3319-3325.

[28] N. Ali, M. Sajid, T. Javed and Z. Abbas, Non-Newtonian flow induced by a peristaltic waves in a curved channel, European J Mech B/Fluids 29 (2010), 387-394. 

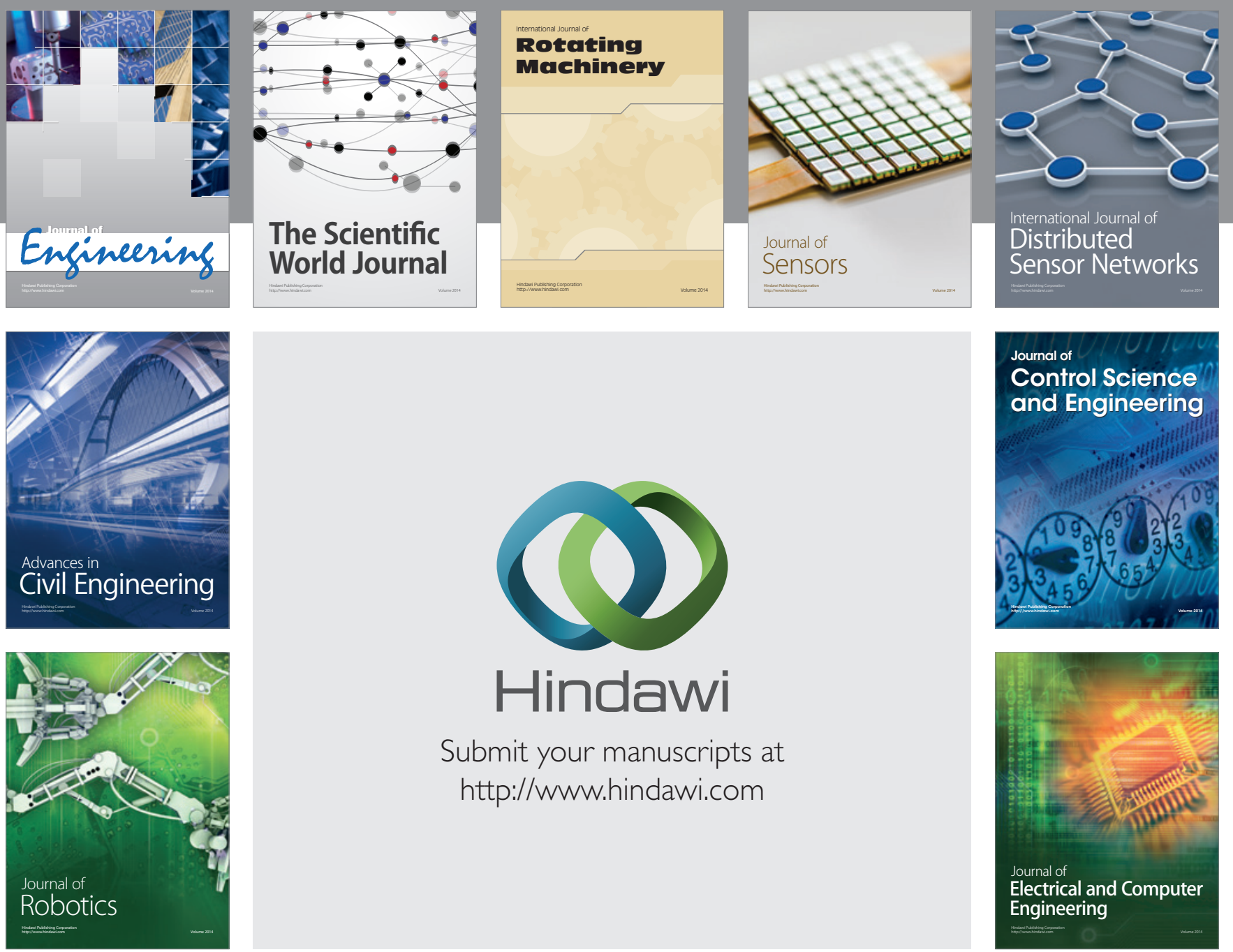

Submit your manuscripts at

http://www.hindawi.com
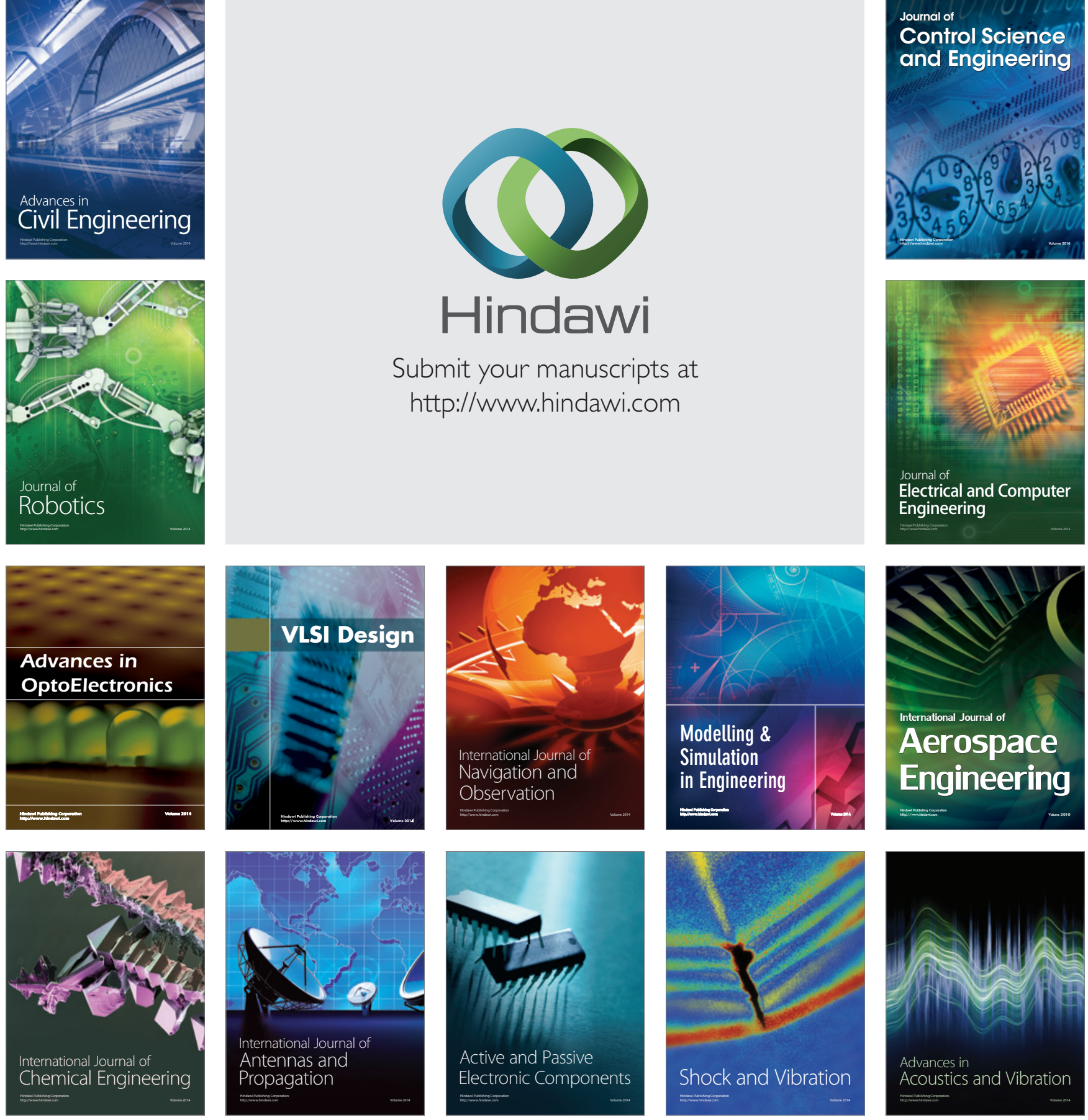